\title{
ON THE GEVREY WELL-POSEDNESS OF THE KIRCHHOFF EQUATION
}

\author{
TOKIO MATSUYAMA AND MICHAEL RUZHANSKY
}

\begin{abstract}
This paper is devoted to proving the almost global solvability of the Cauchy problem for the Kirchhoff equation in the Gevrey space $\gamma_{\eta, L^{2}}^{s}$. Furthermore, similar results are obtained for the initial-boundary value problems in bounded domains and in exterior domains with compact boundary.
\end{abstract}

\section{INTRODUCTION}

G. Kirchhoff proposed the equation

$$
\partial_{t}^{2} u-\left(1+\int_{\Omega}|\nabla u(t, y)|^{2} d y\right) \Delta u=0 \quad(t \in \mathbb{R}, x \in \Omega)
$$

in his book on mathematical physics in 1876, as a model equation for transversal motion of the elastic string, where $\Omega$ is a domain in $\mathbb{R}^{n}$ (see [20], and for finite dimensional approximation problem, see Nishida [30]). Since then, it was first in 1940 that Bernstein proved the existence of global in time analytic solutions on an interval of real line in his celebrated paper [3]. After him, Arosio and Spagnolo discussed analytic solutions in higher spatial dimensions (see [2]), and D'Ancona and Spagnolo proved analytic well-posedness for the degenerate Kirchhoff equation (see [6], and also Kajitani and Yamaguti [19]).

As it is well known, this equation has a Hamiltonian structure, nevertheless it involves a challenging problem whether or not, one can prove the existence of time global solutions corresponding to data in Gevrey classes, $H^{\infty}$-class or standard Sobolev spaces without smallness condition. Up to now, there is no solution to these problems.

The global existence of quasi-analytic solutions is known, see Ghisi and Gobbino, Nishihara, Pohozhaev $([12,31,32])$. Here quasi-analytic classes are intermediate ones between the analytic class and the $C^{\infty}$-class. Manfrin discussed the time global solutions in Sobolev spaces corresponding to non-analytic data having a spectral gap (see [23]), and a similar result is obtained by Hirosawa (see [17]).

On the other hand, global well-posedness in Sobolev space $H^{3 / 2}$, or $H^{2}$ with small data is well established in $[4,7,8,9,14,18,24,25,34,38,39]$. There, the classes of small data consist of compactly supported functions (see [14]), or more generally, they are characterised by some weight conditions (see $[4,7,8,9]$ ) or oscillatory integrals (see $[18,15,24,25,33,34,38,39]$ ). Recently, the authors studied the global wellposedness for Kirchhoff systems with small data (see [28]), and generalised all the

2010 Mathematics Subject Classification. Primary 35L40, 35L30; Secondary 35L10, 35L05, 35L75;

Key words and phrases. Kirchhoff equation; Gevrey class; exterior problem.

The second author was supported in parts by the EPSRC grant EP/K039407/1 and EPSRC Leadership Fellowship EP/G007233/1. 
previous results in the framework of small data. Here, the class of data in [28] consists of Sobolev space $\left(H^{1}\right)^{m}, m$ being the order of system, and is characterised by some oscillatory integrals. The precise statements of the known results can be found in the survey paper [26].

The aim of this paper is to prove the almost global existence of solutions in Gevrey spaces (see Theorem 1.1). Furthermore, we indicate how to modify the proof to also yield the global existence for the initial-boundary value problem in exterior domains, and in bounded domains (see Theorem 4.1 and Theorem 4.2, respectively).

In this paper we consider the Cauchy problem for the Kirchhoff equation

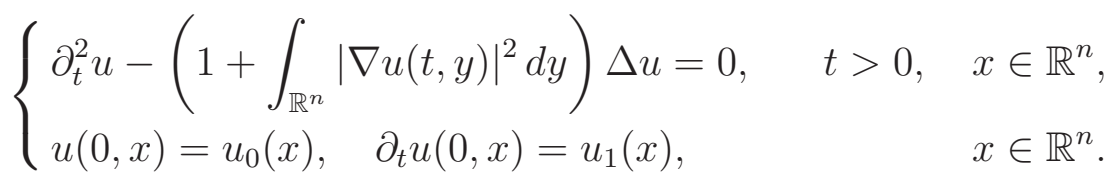

Equation (1.1) has a Hamiltonian structure. More precisely, let us define the energy:

$$
\mathscr{H}(u ; t):=\frac{1}{2}\left\{\|\nabla u(t)\|_{L^{2}}^{2}+\left\|\partial_{t} u(t)\right\|_{L^{2}}^{2}\right\}+\frac{1}{4}\|\nabla u(t)\|_{L^{2}}^{4} .
$$

Then we have

$$
\mathscr{H}(u ; t)=\mathscr{H}(u ; 0)
$$

as long as a solution exists (see Lemma 3.1). We shall now recall the definition of Gevrey class of $L^{2}$ type. For $s \geq 1$, we denote by $\gamma_{L^{2}}^{s}=\gamma_{L^{2}}^{s}\left(\mathbb{R}^{n}\right)$ the Roumieu-Gevrey class of order $s$ on $\mathbb{R}^{n}$ :

$$
\gamma_{L^{2}}^{s}=\bigcup_{\eta>0} \gamma_{\eta, L^{2}}^{s}
$$

where $f$ belong to $\gamma_{\eta, L^{2}}^{s}$ if and only if

$$
\int_{\mathbb{R}^{n}} e^{\eta|\xi|^{1 / s}}|\widehat{f}(\xi)|^{2} d \xi<\infty
$$

where $\widehat{f}(\xi)$ stands for the Fourier transform of $f(x)$. The class $\gamma_{L^{2}}^{s}$ is endowed with the inductive limit topology. In particular, if $s=1$, then $\gamma_{L^{2}}^{1}\left(\mathbb{R}^{n}\right)$ is the class $\mathcal{A}_{L^{2}}$ of the analytic functions on $\mathbb{R}^{n}$. We will use the norms

$$
\|f\|_{\gamma_{\eta, L^{2}}^{s}}=\left[\int_{\mathbb{R}^{n}} e^{\eta|\xi|^{1 / s}}|\widehat{f}(\xi)|^{2} d \xi\right]^{1 / 2}
$$

and

for $\eta>0$.

$$
\|(f, g)\|_{\gamma_{\eta, L^{2}}^{s} \times \gamma_{\eta, L^{2}}^{s}}=\left[\int_{\mathbb{R}^{n}} e^{\eta|\xi|^{1 / s}}\left\{|\widehat{f}(\xi)|^{2}+|\widehat{g}(\xi)|^{2}\right\} d \xi\right]^{1 / 2}
$$

We shall prove here the followings:

Theorem 1.1. Let $T>0$ and $s>1$. Let $M>2, R>0$ and denote $\eta_{0}(M, R, T)=$ $2 s M^{2} e^{4 M^{2}} R T^{1+\frac{1}{s}}+4 M^{2}$. If the functions $u_{0}, u_{1} \in \gamma_{L^{2}}^{s}$, for some $\eta>\eta_{0}(M, R, T)$, satisfy conditions

$$
2 \mathscr{H}(u ; 0)<\frac{M^{2}}{4}-1
$$




$$
\left\|\left((-\Delta)^{3 / 4} u_{0},(-\Delta)^{1 / 4} u_{1}\right)\right\|_{\gamma_{\eta, L^{2}}^{s} \times \gamma_{\eta, L^{2}}^{s}}^{2} \leq R
$$

then the Cauchy problem (1.1) admits a unique solution $u \in C^{1}\left([0, T] ; \gamma_{L^{2}}^{s}\right)$.

We note that Theorem 1.1 does not seem to require the smallness of data. In fact, $M$ and $R$ (measuring the size of the data) are allowed to be large. However, it follows that $\eta$ (measuring the regularity of the data) then also have to be big. So, we can informally describe conditions of Theorem 1.1 that 'the larger the data is the more regular it has to be'.

We can also make the following observation concerning the statement of Theorem 1.1 .

Remark 1.2. The formula for $\eta_{0}(M, R, T)$ in Theorem 1.1 comes from condition (3.6) with s and $q$ related by (3.5). The proof actually yields a more precise conclusion, namely, that the solution $u$ from Theorem 1.1 satisfies

$$
u \in \bigcap_{j=0}^{1} C^{j}\left([0, T] ;(-\Delta)^{-(3 / 4)+(j / 2)} \gamma_{\eta^{\prime}, L^{2}}^{s} \cap(-\Delta)^{-(1 / 2)+(j / 2)} \gamma_{\eta^{\prime}, L^{2}}^{s}\right),
$$

with

$$
\eta^{\prime}=\eta-\eta_{0}(M, R, T)>0 .
$$

This and the order $\eta^{\prime}$ in (1.2) can be found from (3.3) and (3.4) with s and $q$ related by (3.5).

This paper is organised as follows; in $§ 2$ energy estimates for linear equations with time-dependent coefficients will be derived, and these estimates will be applied to get a priori estimates. Sections 3 will be devoted to proof of Theorems 1.1. In $\S 4$

some results on global well-posedness for the initial-boundary value problems will be discussed.

Acknowledgements. The authors would like to express their sincere gratitude to Professors Taeko Yamazaki, Kenji Nishihara, Makoto Nakamura and Doctor Tsukasa Iwabuchi for fruitful discussions. The authors would like to thank also to Professors Kiyoshi Mochizuki, Hiroshi Uesaka and Masaru Yamaguchi for giving them many useful advices.

\section{ENERGy ESTIMATES FOR LINEAR EQUATION}

In this section we shall derive energy estimates for solutions of the linear Cauchy problem with time-dependent coefficients. These estimates will be fundamental tools in the proof of the theorems.

Let us consider the linear Cauchy problem

$$
\left\{\begin{array}{lll}
\partial_{t}^{2} u-c(t)^{2} \Delta u=0, & t \in(0, T), & x \in \mathbb{R}^{n}, \\
u(0, x)=u_{0}(x), \quad \partial_{t} u(0, x)=u_{1}(x), & x \in \mathbb{R}^{n} .
\end{array}\right.
$$

The assumptions for the following estimates are related with Theorem 2 from Colombini, Del Santo and Kinoshita [5]. However, here we need more precise conclusions on the behaviour of constants. 
Proposition 2.1. Let $\sigma \geq 1$ and $1 \leq s<q /(q-1)$ for some $q>1$. Assume that $c=c(t) \in \operatorname{Lip}_{\text {loc }}([0, T))$ satisfies

$$
\begin{gathered}
m_{0} \leq c(t) \leq M, \quad t \in[0, T] \\
\left|c^{\prime}(t)\right| \leq \frac{K}{(T-t)^{q}}, \quad \text { a.e. } t \in[0, T),
\end{gathered}
$$

for some $0<m_{0}<M$ and $K>0$. If $\left((-\Delta)^{\sigma / 2} u_{0},(-\Delta)^{(\sigma-1) / 2} u_{1}\right) \in \gamma_{\eta, L^{2}}^{s} \times \gamma_{\eta, L^{2}}^{s}$ for some $\eta$ satisfying

$$
\eta>\frac{2 K m_{0}^{-1}}{q-1}+4 M^{2} m_{0}^{-1}
$$

then the Cauchy problem (2.1) admits a unique solution

$$
u \in \bigcap_{j=0}^{1} C^{j}\left([0, T] ;(-\Delta)^{-(\sigma-j) / 2} \gamma_{\eta^{\prime}, L^{2}}^{s}\right)
$$

such that

$$
\begin{aligned}
& m_{0}^{2}\left\|(-\Delta)^{\sigma / 2} u(t)\right\|_{\gamma_{\eta^{\prime}, L^{2}}^{s}}^{2}+\left\|(-\Delta)^{(\sigma-1) / 2} \partial_{t} u(t)\right\|_{\gamma_{\eta^{\prime}, L^{2}}^{s}}^{2} \\
\leq & \max \left\{M^{2}, 1\right\} e^{4 M^{2} m_{0}^{-1} \max \left\{1, T^{1-(q s-s)}\right\}}\left\|\left((-\Delta)^{\sigma / 2} u_{0},(-\Delta)^{(\sigma-1) / 2} u_{1}\right)\right\|_{\gamma_{\eta, L^{2}}^{s} \times \gamma_{\eta, L^{2}}^{s}}^{2}
\end{aligned}
$$

for $t \in[0, T]$, where

$$
\eta^{\prime}=\eta-\left(\frac{2 K m_{0}^{-1}}{q-1}+4 M^{2} m_{0}^{-1}\right)>0
$$

Proof. Let $v=v(t, \xi)$ be a solution of the Cauchy problem

$$
\left\{\begin{array}{l}
\partial_{t}^{2} v+c(t)^{2}|\xi|^{2} v=0, \\
v(0, \xi)=\widehat{u}_{0}(\xi), \quad \partial_{t} v(0, \xi)=\widehat{u}_{1}(\xi)
\end{array} \quad t \in(0, T)\right.
$$

We define

$$
c_{*}(t, \xi)= \begin{cases}c(T) & \text { if } T|\xi|^{1 /(q s-s)} \leq 1, \\ c(t) & \text { if } T|\xi|^{1 /(q s-s)}>1 \text { and } 0 \leq t \leq T-|\xi|^{-1 /(q s-s)}, \\ c\left(T-|\xi|^{-1 /(q s-s)}\right) & \text { if } T|\xi|^{1 /(q s-s)}>1 \text { and } T-|\xi|^{-1 /(q s-s)}<t \leq T\end{cases}
$$

and

$$
\alpha(t, \xi)=2 M m_{0}^{-1}\left|c_{*}(t, \xi)-c(t)\right||\xi|+\frac{2\left|c_{*}^{\prime}(t, \xi)\right|}{c_{*}(t, \xi)} .
$$

We adopt an energy for $v$ as

$$
E(t, \xi)=\left\{\left|v^{\prime}(t)\right|^{2}+c_{*}(t, \xi)^{2}|\xi|^{2}|v(t)|^{2}\right\} k(t, \xi),
$$

where

$$
k(t, \xi)=|\xi|^{2(\sigma-1)} \exp \left(-\int_{0}^{t} \alpha(\tau, \xi) d \tau+\eta|\xi|^{1 / s}\right)
$$

and $\eta$ is as in (2.4). We put

$$
\mathcal{E}(t)=\int_{\mathbb{R}^{n}} E(t, \xi) d \xi
$$


Hereafter we concentrate on estimating the integral of $\alpha(t, \xi)$. When $T|\xi|^{1 /(q s-s)} \leq 1$, we can estimate, by using assumption (2.2) on $c(t)$,

$$
\begin{aligned}
\int_{0}^{t} \alpha(\tau, \xi) d \tau & =\int_{0}^{T} 2 M m_{0}^{-1}\left|c_{*}(\tau, \xi)-c(\tau)\right||\xi| d \tau \\
& \leq 4 M^{2} m_{0}^{-1} T|\xi| \\
& \leq 4 M^{2} m_{0}^{-1} T^{1-(q s-s)}
\end{aligned}
$$

and when $T|\xi|^{1 /(q s-s)}>1$, we can estimate

$$
\begin{aligned}
\int_{0}^{t} \alpha(\tau, \xi) d \tau & \leq \int_{0}^{T-|\xi|^{-1 /(q s-s)}} \frac{2\left|c^{\prime}(\tau)\right|}{c(\tau)} d \tau+\int_{T-|\xi|^{-1 /(q s-s)}}^{T} 2 M m_{0}^{-1}\left|c_{*}(\tau, \xi)-c(\tau)\right||\xi| d \tau \\
& \leq \int_{0}^{T-|\xi|^{-1 /(q s-s)}} \frac{2 K m_{0}^{-1}}{(T-\tau)^{q}} d \tau+4 M^{2} m_{0}^{-1}|\xi|^{1-1 /(q s-s)} \\
& \leq \frac{2 K m_{0}^{-1}|\xi|^{1 / s}}{q-1}+4 M^{2} m_{0}^{-1}|\xi|^{1-1 /(q s-s)} .
\end{aligned}
$$

Since $1-1 /(q s-s)<1 / s$, it follows that

$$
|\xi|^{1-1 /(q s-s)} \leq 1+|\xi|^{1 / s}
$$

Consequently, we get

$$
k(t, \xi) \geq e^{-4 M^{2} m_{0}^{-1} \max \left\{1, T^{1-(q s-s)}\right\}}|\xi|^{2(\sigma-1)} e^{\left(\eta-\frac{2 K m_{0}^{-1}}{q-1}-4 M^{2} m_{0}^{-1}\right)|\xi|^{1 / s}},
$$

and hence,

$$
\begin{aligned}
& \mathcal{E}(t) \geq e^{-4 M^{2} m_{0}^{-1} \max \left\{1, T^{1-(q s-s)}\right\}} \times \\
& \int_{\mathbb{R}^{n}} e^{\left(\eta-\frac{2 K m_{0}^{-1}}{q-1}-4 M^{2} m_{0}^{-1}\right)|\xi|^{1 / s}}|\xi|^{2(\sigma-1)}\left\{m_{0}^{2}|\xi|^{2}|v(t)|^{2}+\left|v^{\prime}(t)\right|^{2}\right\} d \xi
\end{aligned}
$$

We compute the derivative of $E(t, \xi)$ :

$$
\begin{aligned}
& E^{\prime}(t, \xi)= \\
& {\left[2 \operatorname{Re}\left\{v^{\prime \prime}(t) \overline{v^{\prime}(t)}\right\}+2 c_{*}(t, \xi) c_{*}^{\prime}(t, \xi)|\xi|^{2}|v(t)|^{2}+2 c_{*}(t, \xi)^{2}|\xi|^{2} \operatorname{Re}\left\{v^{\prime}(t) \overline{v(t)}\right\}\right] k(t, \xi) } \\
& -\left\{c_{*}(t, \xi)^{2}|\xi|^{2}|v(t)|^{2}+\left|v^{\prime}(t)\right|^{2}\right\} \alpha(t, \xi) k(t, \xi) \\
= & {\left[2\left\{c_{*}(t, \xi)^{2}-c(t)^{2}\right\}|\xi|^{2} \operatorname{Re}\left\{v^{\prime}(t) \overline{v(t)}\right\}+2 c_{*}(t, \xi) c_{*}^{\prime}(t, \xi)|\xi|^{2}|v(t)|^{2}\right] k(t, \xi) } \\
& -\alpha(t, \xi) E(t, \xi) .
\end{aligned}
$$


Then we can estimate the right hand side as

$$
\begin{aligned}
& {\left[\frac{2\left|c_{*}(t, \xi)^{2}-c(t)^{2}\right||\xi|}{c_{*}(t, \xi)}\left|v^{\prime}(t)\right| \cdot c_{*}(t, \xi)|\xi||v(t)|+2 \frac{\left|c_{*}^{\prime}(t, \xi)\right|}{c_{*}(t, \xi)} c_{*}(t, \xi)^{2}|\xi|^{2}|v(t)|^{2}\right] k(t, \xi) } \\
& -\alpha(t, \xi) E(t, \xi) \\
\leq & 2 M m_{0}^{-1}\left|c_{*}(t, \xi)-c(t)\right||\xi| E(t, \xi)+\frac{2\left|c_{*}^{\prime}(t, \xi)\right|}{c_{*}(t, \xi)} E(t, \xi)-\alpha(t, \xi) E(t, \xi) \\
= & 0,
\end{aligned}
$$

which implies that $E^{\prime}(t, \xi) \leq 0$ for $t \in(0, T)$, and we find that

$$
\mathcal{E}(t) \leq \mathcal{E}(0)
$$

Thus the required estimate (2.5) follows from this estimate and (2.8). The proof of Proposition 2.1 is now finished.

\section{Proof of Theorem 1.1}

We denote by

$$
H^{\sigma}=H^{\sigma}\left(\mathbb{R}^{n}\right)=(1-\Delta)^{-\sigma / 2} L^{2}\left(\mathbb{R}^{n}\right)
$$

for $\sigma \in \mathbb{R}$ the standard Sobolev spaces, and their homogeneous version is

$$
\dot{H}^{\sigma}=\dot{H}^{\sigma}\left(\mathbb{R}^{n}\right)=(-\Delta)^{-\sigma / 2} L^{2}\left(\mathbb{R}^{n}\right)
$$

Kirchhoff equation has a Hamiltonian structure. Namely, we have:

Lemma 3.1. Let $u \in \bigcap_{j=0}^{1} C^{j}\left(\left[0, T_{u}\right) ; H^{(3 / 2)-j}\right)$ be the solution of (1.1). Then we have

$$
\mathscr{H}(u ; t)=\mathscr{H}(u ; 0), \quad \forall t \in\left[0, T_{u}\right)
$$

where we recall that

$$
\mathscr{H}(u ; t)=\frac{1}{2}\left\{\|\nabla u(t)\|_{L^{2}}^{2}+\left\|\partial_{t} u(t)\right\|_{L^{2}}^{2}\right\}+\frac{1}{4}\|\nabla u(t)\|_{L^{2}}^{4}
$$

Proof. The proof of Lemma 3.1 is elementary. Multiplying equation (1.1) by $\partial_{t} u$ and integrating, we get

as desired.

$$
\frac{d}{d t} \mathscr{H}(u ; t)=0
$$

Now we consider the linear Cauchy problem in the strip $\left(0, T_{u}\left(v_{0}, v_{1}\right)\right) \times \mathbb{R}^{n}$ :

$$
\partial_{t}^{2} v-c(t)^{2} \Delta v=0, \quad t \in(0, T), \quad x \in \mathbb{R}^{n}
$$

with initial condition

$$
v(0, x)=v_{0}(x), \quad \partial_{t} v(0, x)=v_{1}(x) .
$$

Here $c(t)$ belongs to a class $\mathscr{K}$ defined as follows:

Class $\mathscr{K}(T)$. Let $T>0$. Given constants $q>1, M>1$ and $K_{0}>0$, we say that $c(t)$ belongs to $\mathscr{K}(T)=\mathscr{K}\left(q, M, K_{0}, T\right)$ if $c(t)$ belongs to $\operatorname{Lip}_{\text {loc }}([0, T))$ and satisfies

$$
1 \leq c(t) \leq M, \quad t \in[0, T]
$$




$$
\left|c^{\prime}(t)\right| \leq \frac{K_{0}}{(T-t)^{q}}, \quad \text { a.e. } t \in[0, T) .
$$

By the energy estimate (2.5) from Proposition 2.1, there exists a real $\eta>0$ such that if $\left(v_{0}, v_{1}\right) \in(-\Delta)^{-3 / 4} \gamma_{\eta, L^{2}}^{s} \times(-\Delta)^{-1 / 4} \gamma_{\eta, L^{2}}^{s}$, then the Cauchy problem (3.1)-(3.2) admits a unique solution $v$ satisfying

$$
v \in \bigcap_{j=0}^{1} C^{j}\left([0, T] ;(-\Delta)^{-(3 / 4)+(j / 2)} \gamma_{\eta^{\prime}, L^{2}}^{s} \cap(-\Delta)^{-(1 / 2)+(j / 2)} \gamma_{\eta^{\prime}, L^{2}}^{s}\right),
$$

provided that $1 \leq s<q /(q-1)$ and $q>1$, where $\eta^{\prime}>0$ is the real number satisfying

$$
\eta^{\prime}=\eta-\left(\frac{2 K_{0}}{q-1}+4 M^{2}\right)>0 .
$$

If we define the function

$$
\tilde{c}(t):=\sqrt{1+\int_{\mathbb{R}^{n}}|\nabla v(t, x)|^{2} d x},
$$

this defines the mapping

$$
\Theta: c(t) \mapsto \tilde{c}(t)
$$

We will show the compactness of $\mathcal{K}(T)$ in $L_{\text {loc }}^{\infty}([0, T))$ and the continuity of $\Theta$. The convexity of $\mathcal{K}(T)$ is clear. If we show that $\Theta$ maps $\mathcal{K}(T)$ into itself, the SchauderTychonoff fixed point theorem allows us to conclude the proof.

We shall prove here the following:

Proposition 3.2. Let $M>2, T>0$ and $R>0$. Let $1<q<2$ and $s>1$ be such that

$$
s=\frac{1}{q-1}
$$

Let $\eta>0$ be such that

$$
\eta>\frac{2 M^{2} e^{4 M^{2}} R T^{q}}{q-1}+4 M^{2} .
$$

If $\left(v_{0}, v_{1}\right) \in(-\Delta)^{-3 / 4} \gamma_{\eta, L^{2}}^{s} \times(-\Delta)^{-1 / 4} \gamma_{\eta, L^{2}}^{s}$ satisfy

$$
\begin{gathered}
2 \mathscr{H}(v ; 0) \leq \frac{M^{2}}{4}-1, \\
\left\|\left((-\Delta)^{3 / 4} v_{0},(-\Delta)^{1 / 4} v_{1}\right)\right\|_{\gamma_{\eta, L^{2}}^{s} \times \gamma_{\eta, L^{2}}^{s}}^{2} \leq R,
\end{gathered}
$$

then, setting

$$
K_{0}=M^{2} e^{4 M^{2}} R T^{q},
$$

we have the following statement: For any $c(t) \in \mathscr{K}(T)$, let $v$ be a solution to the Cauchy problem (3.1)-(3.2) satisfying (3.3). Then

$$
1 \leq \tilde{c}(t) \leq M, \quad t \in[0, T]
$$




$$
\left|\tilde{c}^{\prime}(t)\right| \leq \frac{K_{0}}{(T-t)^{q}}, \quad t \in[0, T) .
$$

Proof. First, we prove (3.11). To this end, we have only to show that

$$
\left|\tilde{c}^{\prime}(t)\right| \leq \frac{K_{0}}{T^{q}}
$$

for $t \in[0, T]$, since the right hand side of (3.12) is bounded by $K_{0} /(T-t)^{q}$ for $t \in[0, T)$. One can readily see that

$$
2 \tilde{c}(t) \tilde{c}^{\prime}(t)=2 \operatorname{Re}\left((-\Delta)^{3 / 4} v(t),(-\Delta)^{1 / 4} \partial_{t} v(t)\right)_{L^{2}},
$$

and hence, we have

$$
\begin{aligned}
\left|\tilde{c}^{\prime}(t)\right| & \leq\|v(t)\|_{\dot{H}^{3 / 2}}\left\|\partial_{t} v(t)\right\|_{\dot{H}^{1 / 2}} \\
& \leq\left\|(-\Delta)^{3 / 4} v(t)\right\|_{\gamma_{\eta^{\prime}, L^{2}}^{s}}\left\|(-\Delta)^{1 / 4} \partial_{t} v(t)\right\|_{\gamma_{\eta^{\prime}, L^{2}}^{s}}
\end{aligned}
$$

for any $\eta^{\prime}>0$, since $\tilde{c}(t) \geq 1$. Then, by the definition (3.9) of $K_{0}$, the constant $\eta$ satisfies the following inequality:

$$
\eta>\frac{2 K_{0}}{q-1}+4 M^{2}
$$

Hence, if $\eta^{\prime}$ is chosen as in (3.4), then, applying the energy estimate (2.5) from Proposition 2.1 to the right hand side of (3.13), we can write

$$
\left|\tilde{c}^{\prime}(t)\right| \leq M^{2} e^{4 M^{2} \max \left\{1, T^{1-(q s-s)}\right\}}\left\|\left((-\Delta)^{3 / 4} v_{0},(-\Delta)^{1 / 4} v_{1}\right)\right\|_{\gamma_{\eta, L^{2}}^{s} \times \gamma_{\eta, L^{2}}^{s}}^{2}
$$

for $t \in[0, T]$. Since $1-(q s-s)=0$ by assumption (3.5), it follows that

$$
e^{4 M^{2} \max \left\{1, T^{1-(q s-s)}\right\}}=e^{4 M^{2}} .
$$

Hence, recalling the definition (3.9) of $K_{0}$ and using (3.8), we conclude from (3.14)(3.15) that

$$
\begin{aligned}
\left|\tilde{c}^{\prime}(t)\right| \leq M^{2} e^{4 M^{2}}\left\|\left((-\Delta)^{3 / 4} v_{0},(-\Delta)^{1 / 4} v_{1}\right)\right\|_{\gamma_{\eta, L^{2}}^{s} \times \gamma_{\eta, L^{2}}^{s}}^{2} & \\
& \leq M^{2} e^{4 M^{2}} \cdot R T^{q} \cdot \frac{1}{T^{q}}=\frac{K_{0}}{T^{q}}
\end{aligned}
$$

for $t \in[0, T]$. Thus we get the required estimate (3.12).

Finally we prove (3.10). In this case, we will not use the energy estimate (2.5) from Proposition 2.1. Our assumption (3.7) implies that

$$
1 \leq \tilde{c}(0) \leq \sqrt{1+2 \mathscr{H}(v ; 0)} \leq \frac{M}{2} .
$$

Since $\tilde{c}(t)$ is continuous, there exists a time $t_{1}<T$ such that

$$
1 \leq \tilde{c}(t) \leq M
$$

for $0 \leq t \leq t_{1}$. Fixing data $\left(v_{0}, v_{1}\right)$ satisfying (3.7)-(3.8), we can show that the class $\mathcal{K}\left(t_{1}, K_{0}\right)$ is the convex and compact subset of the Banach space $L^{\infty}\left(\left[0, t_{1}\right]\right)$, and resorting to (3.11), we can also prove that $\Theta$ is continuous from $\mathcal{K}\left(t_{1}, K_{0}\right)$ into itself. This argument will be also done in the whole interval $[0, T]$ in the last step, 
where we give its details. Then Schauder's fixed point theorem allows us to conclude that $\Theta$ has a fixed point in $\mathcal{K}\left(t_{1}, K_{0}\right)$ :

$$
c(t)=\Theta(c(t))=\tilde{c}(t)
$$

for $0 \leq t \leq t_{1}$. This means that solution $v(t, x)$ to the linear Cauchy problem (3.1)(3.2) is also a solution to the nonlinear Cauchy problem (1.1) with data $\left(v_{0}, v_{1}\right)$ on $\left[0, t_{1}\right]$. Hence it follows from Lemma 3.1 and assumption (3.7) that

$$
2 \mathscr{H}(v ; t)=2 \mathscr{H}(v ; 0) \leq \frac{M^{2}}{4}-1, \quad t \in\left[0, t_{1}\right],
$$

and as a result, we deduce that

$$
1 \leq \tilde{c}(t) \leq \sqrt{1+2 \mathscr{H}(v ; t)} \leq \frac{M}{2}
$$

for $0 \leq t \leq t_{1}$. Therefore, by the continuity of $\tilde{c}(t)$, there exists a time $t_{2} \in\left(t_{1}, T\right)$ such that

$$
1 \leq \tilde{c}(t) \leq M
$$

for $0 \leq t \leq t_{2}$. Hence, we can develop the previous fixed point argument; the solution $v(t, x)$ to the linear Cauchy problem (3.1)-(3.2) is also a solution to the nonlinear Cauchy problem $(1.1)$ with data $\left(v_{0}, v_{1}\right)$ on $\left[0, t_{2}\right]$ satisfying

$$
2 \mathscr{H}(v ; t)=2 \mathscr{H}(v ; 0) \leq \frac{M^{2}}{4}-1, \quad t \in\left[0, t_{2}\right],
$$

where we have used assumption (3.7) in the last step. Now, we define a time $t_{*}$ by the maximal time such that

$$
1 \leq \tilde{c}(t) \leq M
$$

for $0 \leq t \leq t_{*}$. Suppose that $t_{*}<T$. Then, after employing the fixed point argument on the interval $\left[0, t_{*}\right]$, we deduce from Lemma 3.1 and assumption (3.7) that

$$
2 \mathscr{H}\left(v ; t_{*}\right)=2 \mathscr{H}(v ; 0) \leq \frac{M^{2}}{4}-1,
$$

and hence, we get

$$
1 \leq \tilde{c}\left(t_{*}\right) \leq \frac{M}{2}
$$

Therefore, the fixed point argument will be also applicable, and $v(t, x)$ coincides with the solution to $(1.1)$ with data $\left(v_{0}, v_{1}\right)$ on some interval $\left[0, t_{* *}\right]$ strictly containing $\left[0, t_{*}\right]$. This implies that $\tilde{c}(t)$ is bounded by $M$ on $\left[0, t_{* *}\right]$. But this contradicts the maximality of $t_{*}$. Thus we must have the required estimate (3.10). The proof of Proposition 3.2 is now complete.

Based on the previous proposition, we prove our theorem.

Completion of proof of Theorem 1.1. Hereafter, we write

$$
\mathcal{K}=\mathcal{K}(T) .
$$

Let $c(t) \in \mathscr{K}$, we fix the data

$$
\left(v_{0}, v_{1}\right) \in(-\Delta)^{-3 / 4} \gamma_{\eta, L^{2}}^{s} \times(-\Delta)^{-1 / 4} \gamma_{\eta, L^{2}}^{s}
$$


satisfying (3.7)-(3.8). Then it follows from Proposition 3.2 that the mapping

$$
\Theta: c(t) \mapsto \tilde{c}(t)
$$

maps from $\mathscr{K}$ into itself. Now $\mathscr{K}$ may be regarded as the convex subset of the Fréchet space $L_{\text {loc }}^{\infty}([0, T))$, and we endow $\mathscr{K}$ with the induced topology. We shall prove the compactness of $\mathscr{K}$ and continuity of the mapping $\Theta$. Then the Schauder-Tychonoff theorem allows us to conclude the proof.

Compactness of $\mathscr{K}$. We show that $\mathscr{K}$ is uniformly bounded and equi-continuous on every compact interval of $[0, T)$. Let $\left\{c_{k}(t)\right\}_{k=1}^{\infty}$ be a sequence in $\mathscr{K}$ such that

$$
\begin{gathered}
1 \leq c_{k}(t) \leq M, \quad t \in[0, T], \\
\left|c_{k}^{\prime}(t)\right| \leq \frac{K_{0}}{(T-t)^{q}}, \quad \text { a.e. } t \in[0, T) .
\end{gathered}
$$

Observing

$$
c_{k}(t)-c_{k}\left(t^{\prime}\right)=\int_{t^{\prime}}^{t} c_{k}^{\prime}(\tau) d \tau
$$

we obtain from (3.18) that

$$
\left|c_{k}(t)-c_{k}\left(t^{\prime}\right)\right| \leq \frac{K_{0}}{q-1}\left\{\frac{1}{\left(T-t^{\prime}\right)^{q-1}}-\frac{1}{(T-t)^{q-1}}\right\}
$$

for $0 \leq t^{\prime}<t<T$. Since $1 /(T-t)^{q-1}$ is uniformly continuous on every compact interval of $[0, T)$, the sequence $\left\{c_{k}(t)\right\}_{k=1}^{\infty}$ is equi-continuous on that interval. Thus $\mathscr{K}$ is relatively compact in $L_{\text {loc }}^{\infty}([0, T))$, and hence, one can deduce from the ArzelàAscoli theorem that every sequence $\left\{c_{k}(t)\right\}_{k=1}^{\infty}$ in $\mathscr{K}$ has a subsequence, denoted by the same, converging to some $c(\cdot) \in L_{\mathrm{loc}}^{\infty}([0, T))$ :

$$
\left\{\begin{array}{l}
c_{k}(t) \underset{(k \rightarrow \infty)}{\rightarrow} c(t) \quad \text { in } L_{\mathrm{loc}}^{\infty}([0, T)) ; \\
1 \leq c(t) \leq M \quad \text { for every compact interval in }[0, T) ; \\
\left|c(t)-c\left(t^{\prime}\right)\right| \leq \frac{K_{0}}{q-1}\left\{\frac{1}{\left(T-t^{\prime}\right)^{q-1}}-\frac{1}{(T-t)^{q-1}}\right\}, \quad 0 \leq t^{\prime}<t<T .
\end{array}\right.
$$

The last statement of (3.19) implies that $c(t)$ is in $\operatorname{Lip}_{\text {loc }}([0, T))$, since the function $(T-t)^{-(q-1)}$ is in $\operatorname{Lip}_{\text {loc }}([0, T))$. Furthermore, $c(t)$ must be bounded by $M$ even at $t=T$ :

$$
1 \leq c(t) \leq M, \quad t \in[0, T]
$$

Indeed, if

$$
\varlimsup_{t \nearrow T} c(t)>M,
$$

there exists a sequence $\left\{t_{j}\right\}$ such that

$$
t_{j} \nearrow T \text { and } c\left(t_{j}\right)>M, \quad(j=1,2, \ldots) .
$$

Going back to (3.17), and resorting to the first statement of (3.19), we have

$$
c\left(t_{j}\right)=\lim _{k \rightarrow \infty} c_{k}\left(t_{j}\right) \leq M, \quad(\forall j)
$$


which leads to a contradiction. Thus we conclude that $c(t)$ satisfies (3.20) and

$$
c(\cdot) \in \operatorname{Lip}_{\text {loc }}([0, T))
$$

and the derivative $c^{\prime}(t)$ exists a.e. $t \in[0, T)$. Now, for the derivative $c^{\prime}(t)$, if we prove that

$$
\left|c^{\prime}(t)\right| \leq \frac{K_{0}}{(T-t)^{q}}, \quad \text { a.e. } t \in[0, T),
$$

then $c(t) \in \mathscr{K}$, which proves the compactness of $\mathscr{K}$. We prove $(3.21)$. Let $t_{0} \in(0, T)$ be an arbitrary point where $c(t)$ is differentiable. Since we have, by using (3.18),

$$
\begin{array}{r}
\quad\left|\frac{1}{2 h}\left\{c_{k}\left(t_{0}+h\right)-c_{k}\left(t_{0}-h\right)\right\}\right|=\left|\frac{1}{2 h} \int_{t_{0}-h}^{t_{0}+h} c_{k}^{\prime}(t) d t\right| \\
\leq \frac{K_{0}}{2 h(q-1)}\left\{\frac{1}{\left(T-\left(t_{0}-h\right)\right)^{q-1}}-\frac{1}{\left(T-\left(t_{0}+h\right)\right)^{q-1}}\right\}
\end{array}
$$

for $h>0$, we can take the limit in this equation with respect to $k$, so that

$$
\left|\frac{1}{2 h}\left\{c\left(t_{0}+h\right)-c\left(t_{0}-h\right)\right\}\right| \leq \frac{K_{0}}{2 h(q-1)}\left\{\frac{1}{\left(T-\left(t_{0}-h\right)\right)^{q-1}}-\frac{1}{\left(T-\left(t_{0}+h\right)\right)^{q-1}}\right\} .
$$

Then, letting $h \rightarrow+0$, we conclude that

$$
\left|c^{\prime}\left(t_{0}\right)\right| \leq \frac{K_{0}}{\left(T-t_{0}\right)^{q}}
$$

Since $t_{0}$ is arbitrary, we get (3.21).

Continuity of $\Theta$ on $\mathscr{K}$. Let us take a sequence $\left\{c_{k}(t)\right\}$ in $\mathscr{K}$ such that

$$
c_{k}(\cdot) \rightarrow c(\cdot) \in \mathscr{K} \quad \text { in } L_{\mathrm{loc}}^{\infty}([0, T)) \quad(k \rightarrow \infty),
$$

and let $v_{k}(t, x)$ and $v(t, x)$ be corresponding solutions to the linear Cauchy problem (3.1)-(3.2) with coefficients $c_{k}(t)$ and $c(t)$, respectively, with fixed data $\left(v_{0}, v_{1}\right)$. Then it is sufficient to prove that the images $\tilde{c}_{k}(t):=\Theta\left(c_{k}(t)\right)$ and $\tilde{c}(t):=\Theta(c(t))$ satisfy

$$
\tilde{c}_{k}(\cdot) \rightarrow \tilde{c}(\cdot) \quad \text { in } L_{\mathrm{loc}}^{\infty}([0, T)) \quad(k \rightarrow \infty) .
$$

The functions $w_{k}:=v_{k}-v, k=1,2, \ldots$, solve the following Cauchy problem:

$$
\left\{\begin{array}{l}
\partial_{t}^{2} w_{k}-c(t)^{2} \Delta w_{k}=\left\{c_{k}(t)^{2}-c(t)^{2}\right\} \Delta v_{k}, \quad(t, x) \in(0, T) \times \mathbb{R}^{n}, \\
w_{k}(0, x)=0, \quad \partial_{t} w_{k}(0, x)=0, \quad x \in \mathbb{R}^{n} .
\end{array}\right.
$$

If we differentiate the energy $\mathscr{E}\left(w_{k}(t)\right)$ for $w_{k}$ with respect to $t$, where

$$
\mathscr{E}\left(w_{k}(t)\right)=\left\|\partial_{t} w_{k}(t)\right\|_{L^{2}}^{2}+c(t)^{2}\left\|\nabla w_{k}(t)\right\|_{L^{2}}^{2},
$$

we get

$$
\begin{aligned}
\mathscr{E}^{\prime}\left(w_{k}(t)\right)= & -2\left\{c_{k}(t)^{2}-c(t)^{2}\right\} \operatorname{Re}\left(\Delta v_{k}(t), \partial_{t} w_{k}(t)\right)_{L^{2}} \\
& +2 c(t) c^{\prime}(t)\left\|\nabla w_{k}(t)\right\|_{L^{2}}^{2} \\
\leq & 2\left|c_{k}(t)^{2}-c(t)^{2}\right|\left\|v_{k}(t)\right\|_{\dot{H}^{3 / 2}}\left\|\partial_{t} w_{k}(t)\right\|_{\dot{H}^{1 / 2}}+2 \frac{\left|c^{\prime}(t)\right|}{c(t)} \mathscr{E}\left(w_{k}(t)\right) .
\end{aligned}
$$


Here, we see from (2.5) in Proposition 2.1 that

$$
\begin{aligned}
& \left\|v_{k}(t)\right\|_{\dot{H}^{3 / 2}}\left\|\partial_{t} w_{k}(t)\right\|_{\dot{H}^{1 / 2}} \\
\leq & M^{2} e^{4 M^{2} T^{1-(q s-s)}}\left\|\left((-\Delta)^{3 / 2} v_{0},(-\Delta)^{1 / 2} v_{1}\right)\right\|_{\gamma_{\eta, L^{2}}^{s} \times \gamma_{\eta, L^{2}}^{s}}^{2}
\end{aligned}
$$

for $0 \leq t \leq T$. Then we integrate (3.23) and apply Gronwall's lemma to obtain

$$
\begin{aligned}
\mathscr{E}\left(w_{k}(t)\right) \leq M^{2} e^{4 M^{2} m_{0}^{-1} T^{1-(q s-s)}} \| & \left((-\Delta)^{3 / 2} v_{0},(-\Delta)^{1 / 2} v_{1}\right) \|_{\gamma_{\eta, L^{2}}^{s} \times \gamma_{\eta, L^{2}}^{s}}^{2} \times \\
& \left(\int_{0}^{t}\left|c_{k}(\tau)^{2}-c(\tau)^{2}\right| d \tau\right) \exp \left(2 \int_{0}^{t} \frac{\left|c^{\prime}(\tau)\right|}{c(\tau)} d \tau\right)
\end{aligned}
$$

for $t \in[0, T)$, which implies that

$$
\left.\begin{array}{l}
\nabla v_{k}(t) \rightarrow \nabla v(t) \\
\partial_{t} v_{k}(t) \rightarrow \partial_{t} v(t)
\end{array}\right\} \quad \text { in } L_{\mathrm{loc}}^{\infty}\left([0, T) ; L^{2}\right) \text { as } k \rightarrow \infty .
$$

Hence we get (3.22), which proves the continuity of $\Theta$.

We are now in a position to conclude the proof. Proposition 3.2 and the previous results assure that $\Theta$ is continuous from $\mathscr{K}$ into itself, provided that the data $\left(v_{0}, v_{1}\right)$ satisfy (3.7)-(3.8). Since $\mathscr{K}$ is the convex and compact subset of the Fréchet space $L_{\text {loc }}^{\infty}([0, T))$, the Schauder-Tychonoff theorem implies that $\Theta$ has a fixed point in $\mathscr{K}$, and hence, we conclude that solution $v(t, x)$ to the linear Cauchy problem (3.1)-(3.2) is also a solution to the nonlinear Cauchy problem (1.1) with data $\left(v_{0}, v_{1}\right)$ on $[0, T]$.

In conclusion, we obtain that there exist $M>2$ and $R>0$ such that for every $\eta_{0}(M, R, T)>0$ there exists $\eta>\eta_{0}(M, R, T)$ so that if $s>1$ and $\left(u_{0}, u_{1}\right) \in \gamma_{\eta, L^{2}}^{s} \times$ $\gamma_{\eta, L^{2}}^{s}$ satisfy (3.7)-(3.8), the Cauchy problem (1.1) admits a solution $u$ in the class $C^{1}\left([0, \infty) ; \gamma_{L^{2}}^{s}\right)$. The uniqueness is proved by the same argument as in the fixed point one. The proof of Theorem 1.1 is finished.

\section{Initial-BOUndary VAlue PROBlems For the KirchHoff EQUATION}

The argument in the proof of Theorem 1.1 is available for the initial-boundary value problems in an open set $\Omega$ of $\mathbb{R}^{n}$. In this section we discuss the global wellposedness for initial-boundary value problem to the Kirchhoff equation in the typical domains: bounded domains and exterior domains. The results in this section can be proved by Fourier series expansions method in bounded domains, and generalised Fourier transform method in exterior domains, respectively. It is known from spectral theorem that a self-adjoint operator on a separable Hilbert space is unitary equivalent to a multiplication operator on some $L^{2}(\mathcal{M}, \mu)$, where $(\mathcal{M}, \mu)$ is a measure space. Then $L^{2}(\Omega)$ is unitary equivalent to $L^{2}\left(\mathbb{R}^{n}\right)$. This means that the Fourier transform method in $\mathbb{R}^{n}$ is available for $L^{2}$ space on an open set $\Omega$ in $\mathbb{R}^{n}$; any multiplier acting on $L^{2}\left(\mathbb{R}^{n}\right)$ is unitarily transformed into an multiplier acting on $L^{2}(\Omega)$.

4.1. The case: $\Omega$ is an exterior domain. Replacing the Fourier transform over $\mathbb{R}^{n}$ by the generalised Fourier transform over exterior domains and applying exactly 
the same argument of Theorem 1.1, we can also prove a similar result on the initialboundary value problem in exterior domains. More precisely, we consider the following problem:

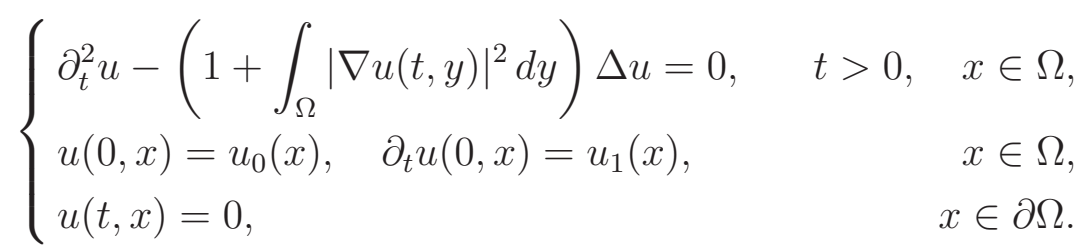

Here, $\Omega$ is a domain in $\mathbb{R}^{n}$ such that $\mathbb{R}^{n} \backslash \Omega$ is compact and its boundary $\partial \Omega$ is analytic. The latter assumption may be in principle relaxed but this would require an extension of known analytic solvability results to the Gevrey setting, so we omit it for this moment, and refer to [1] and [21] for further details.

Following Wilcox [36], let us define the generalised Fourier transforms in an arbitrary exterior domain $\Omega$. Let $A$ be a self-adjoint realisation of the Dirichlet Laplacian $-\Delta$ with domain $H^{2}(\Omega) \cap H_{0}^{1}(\Omega)$. Then $A$ is non-negative on $L^{2}(\Omega)$, and we can define the square root $A^{1 / 2}$ of $A$. We recall the resolvent operator $R\left(|\xi|^{2} \pm i 0\right)$ :

$$
R\left(|\xi|^{2} \pm i 0\right)=\lim _{\varepsilon \rightarrow+0}\left(A-\left(|\xi|^{2} \pm i \varepsilon\right)\right)^{-1}
$$

and $R\left(|\xi|^{2} \pm i 0\right)$ is bounded from $L^{2}\left(\Omega,\langle x\rangle^{s} d x\right)$ to $H^{2}\left(\Omega,\langle x\rangle^{-s} d x\right)$ for each $\xi \in \mathbb{R}^{n}$ and some $s>1 / 2$, where $\langle x\rangle=(1+|x|)^{1 / 2}$ (see, e.g., Mochizuki [29]). Introducing a function $j=j(x) \in C^{\infty}\left(\mathbb{R}^{n}\right)$ vanishing in a neighbourhood of $\mathbb{R}^{n} \backslash \Omega$ and equal to one for large $|x|$, let us define the generalised Fourier transforms as follows:

$$
\left(\mathscr{F}_{ \pm} f\right)(\xi)=\lim _{L \rightarrow \infty}(2 \pi)^{-n / 2} \int_{\Omega \cap\{|x|<L\}} \overline{\psi_{ \pm}(x, \xi)} f(x) d x \quad \text { in } \quad L^{2}\left(\mathbb{R}^{n}\right)
$$

where we put

$$
\begin{gathered}
\psi_{ \pm}(x, \xi)=j(x) e^{i x \cdot \xi}+\left[R\left(|\xi|^{2} \pm i 0\right) M_{\xi}(\cdot)\right](x) \\
\text { with } M_{\xi}(x)=-\left(A-|\xi|^{2}\right)\left(j(x) e^{i x \cdot \xi}\right) .
\end{gathered}
$$

Notice that we can write formally

$$
M_{\xi}(x)=\{\Delta j(x)+2 i \xi \cdot \nabla j(x)\} e^{i x \cdot \xi} .
$$

The kernels $\psi_{ \pm}(x, \xi)$ are called eigenfunctions of the operator $A$ with eigenvalue $|\xi|^{2}$ in the sense that, formally,

$$
\left(A-|\xi|^{2}\right) \psi_{ \pm}(x, \xi)=0
$$

but $\psi_{ \pm}(x, \xi) \notin L^{2}(\Omega)$. Similarly, the inverse transforms are defined by

$$
\left(\mathscr{F}_{ \pm}^{*} g\right)(x)=\lim _{L \rightarrow \infty}(2 \pi)^{-n / 2} \int_{\{|\xi|<L\}} \psi_{ \pm}(x, \xi) g(\xi) d \xi \quad \text { in } L^{2}(\Omega) .
$$

We treat $\mathscr{F}_{+} f$ only and drop the subscript + , since $\mathscr{F}_{-} f$ can be dealt with by essentially the same method. The transform $\mathscr{F} f$ thus defined obeys the following properties (see, e.g., Shenk II [35, Theorem 1 and Corollary 5.1]):

(i) $\mathscr{F}$ is a unitary mapping

$$
\mathscr{F}: L^{2}(\Omega) \rightarrow L^{2}\left(\mathbb{R}^{n}\right) .
$$


Hence

$$
\mathscr{F} \mathscr{F}^{*}=I \text {. }
$$

(ii) $\mathscr{F}$ satisfies the generalised Parseval identity:

$$
(\mathscr{F} f, \mathscr{F} g)_{L^{2}\left(\mathbb{R}^{n}\right)}=(f, g)_{L^{2}(\Omega)}, \quad f, g \in L^{2}(\Omega) .
$$

(iii) $\mathscr{F}$ diagonalises the operator $A$ in the sense that

$$
\mathscr{F}(\varphi(A) f)(\xi)=\varphi\left(|\xi|^{2}\right)(\mathscr{F} f)(\xi),
$$

where $\varphi(A)$ is the operator defined by the spectral representation theorem for self-adjoint operators.

We define the Sobolev spaces over $\Omega$ as follows:

$$
H^{\sigma}(\Omega)=(1+A)^{-\sigma / 2} L^{2}(\Omega) \quad \text { for } \sigma \in \mathbb{R},
$$

and their homogeneous version is defined as

$$
\dot{H}^{\sigma}(\Omega)=A^{-\sigma / 2} L^{2}(\Omega) \quad \text { for } \sigma \in \mathbb{R} .
$$

We say that $f \in \gamma_{L^{2}}^{s}(\Omega)$ for $s \geq 1$ if and only if there exists a constant $\eta>0$ such that

$$
\int_{\mathbb{R}^{n}} e^{\eta|\xi|^{1 / s}}|(\mathscr{F} f)(\xi)|^{2} d \xi<\infty
$$

The class $\gamma_{L^{2}}^{s}(\Omega)$ is endowed with the projective limit topology.

To state the results, we need to introduce the analytic compatibility condition.

The Gevrey compatibility condition. $f$ satisfies the Gevrey compatibility condition if and only if $f \in \gamma_{L^{2}}^{s}(\Omega)$ satisfies

$$
A^{k} f \in H_{0}^{1}(\Omega), \quad k=0,1, \cdots .
$$

Based on the properties (i)-(iii) of the generalised Fourier transform, we have:

Theorem 4.1. Assume that $\Omega$ is an exterior domain of $\mathbb{R}^{n}$ such that $\mathbb{R}^{n} \backslash \Omega$ is compact with analytic boundary $\partial \Omega$. Let $T>0$ and $s>1$. Suppose that there exist $M>2, R>0$ and $\eta>\eta_{0}(M, R, T)$ for a sufficiently large $\eta_{0}(M, R, T)$ such that the functions $u_{0}, u_{1} \in \gamma_{L^{2}}^{s}(\Omega)$ satisfy

$$
\begin{gathered}
2 \mathscr{H}(u ; 0)<\frac{M^{2}}{4}-1, \\
\left\|\left((-\Delta)^{3 / 4} u_{0},(-\Delta)^{1 / 4} u_{1}\right)\right\|_{\gamma_{\eta, L^{2}}^{s} \times \gamma_{\eta, L^{2}}^{s}}^{2} \leq R .
\end{gathered}
$$

Then the initial-boundary-value problem (4.1) admits a unique solution $u$ in the class

$$
C^{1}\left([0, \infty) ; \gamma_{L^{2}}^{s}(\Omega)\right)
$$

The constants can be made precise, in the same way as in Remark 1.2. 
4.2. The case: $\Omega$ is a bounded domain. Replacing Fourier transform by Fourier series expansion and applying exactly the same argument of the proof of Theorem 1.1 , we can prove a similar result for the initial-boundary value problem in $[0, \infty) \times \Omega$, where $\Omega$ is a bounded domain in $\mathbb{R}^{n}$ with analytic boundary $\partial \Omega$. Let $\left\{w_{k}\right\}_{k=1}^{\infty}$ be a complete orthonormal system of eigenfunctions of the operator $-\Delta$ whose domain is $H^{2}(\Omega) \cap H_{0}^{1}(\Omega)$, and let $\lambda_{k}$ be eigenvalues corresponding to $w_{k}$. Namely, $\left\{w_{k}, \lambda_{k}\right\}$ satisfy the elliptic equations:

$$
\left\{\begin{aligned}
-\Delta w_{k} & =\lambda_{k} w_{k} & & \text { in } \Omega, \\
w_{k} & =0 & & \text { on } \partial \Omega .
\end{aligned}\right.
$$

Then $\left(w_{k}, w_{\ell}\right)_{L^{2}(\Omega)}=\delta_{k \ell}$ and

$$
0<\lambda_{1} \leq \lambda_{2} \leq \cdots \leq \lambda_{k} \leq \cdots \text { and } \lambda_{k} \rightarrow \infty,
$$

where $(\phi, \psi)_{L^{2}(\Omega)}$ stands for the inner product of $\phi$ and $\psi$ in $L^{2}(\Omega)$. We say that $f \in H^{\sigma}(\Omega)$ for real $\sigma$ if

$$
\sum_{k=1}^{\infty} \lambda_{k}^{2 \sigma}\left|\left(f, w_{k}\right)_{L^{2}(\Omega)}\right|^{2}<\infty,
$$

and $f \in \gamma_{L^{2}}^{s}(\Omega)$ for $s \geq 1$ if and only if there exists a constant $\eta>0$ such that

$$
\sum_{k=1}^{\infty} e^{\eta \lambda_{k}^{1 / s}}\left|\left(f, w_{k}\right)_{L^{2}(\Omega)}\right|^{2}<\infty .
$$

The class $\gamma_{L^{2}}^{s}(\Omega)$ is endowed with the inductive limit topology.

Then we have:

Theorem 4.2. Assume that $\Omega$ is a bounded domain in $\mathbb{R}^{n}$ with analytic boundary $\partial \Omega$. Let $T>0$ and $s>1$. Suppose that there exist $M>2, R>0$ and $\eta>\eta_{0}(M, R, T)$ for a sufficiently large $\eta_{0}(M, R, T)$ such that the functions $u_{0}, u_{1} \in \gamma_{L^{2}}^{s}(\Omega)$ satisfy

$$
\begin{gathered}
2 \mathscr{H}(u ; 0)<\frac{M^{2}}{4}-1, \\
\left\|\left((-\Delta)^{3 / 4} u_{0},(-\Delta)^{1 / 4} u_{1}\right)\right\|_{\gamma_{\eta, L^{2}}^{s} \times \gamma_{\eta, L^{2}}^{s}}^{2} \leq R .
\end{gathered}
$$

Then the initial-boundary-value problem (4.1) admits a unique solution $u$ in the class

$$
C^{1}\left([0, \infty) ; \gamma_{L^{2}}^{s}(\Omega)\right) \text {. }
$$

Again, the constants can be made precise, in the same way as in Remark 1.2.

\section{REFERENCES}

[1] A. Arosio, Asymptotic behaviour as $t \rightarrow+\infty$ of the solutions of linear hyperbolic equations with coefficients discontinuous in time (on a bounded domain), J. Differential Equations 39 (1981), 291-309.

[2] A. Arosio and S. Spagnolo, Global solutions to the Cauchy problem for a nonlinear hyperbolic equation, Nonlinear partial differential equations and their applications, Collège de France seminar, Vol. VI (Paris, 1982/1983), pp. 1-26, Res. Notes in Math., 109, Pitman, Boston, MA, 1984.

[3] S. Bernstein, Sur une classe d'équations fonctionnelles aux dérivées partielles, Izv. Akad. Nauk SSSR Ser. Mat. 4 (1940), 17-27. 
[4] E. Callegari and R. Manfrin, Global existence for nonlinear hyperbolic systems of Kirchhoff type, J. Differential Equations 132 (1996), 239-274.

[5] F. Colombini, D. Del Santo and T. Kinoshita, Well-posedness of a hyperbolic equation with non-Lipschitz coefficients, Ann. Scuola Norm. Sup. Pisa, Cl. Sci. (5), 1 (2002), 327-358.

[6] P. D'Ancona and S. Spagnolo, Global solvability for the degenerate Kirchhoff equation with real analytic data, Invent. Math. 108 (1992), 247-262.

[7] P. D'Ancona and S. Spagnolo, A class of nonlinear hyperbolic problems with global solutions, Arch. Rational Mech. Anal. 124 (1993), 201-219.

[8] P. D'Ancona and S. Spagnolo, Nonlinear perturbations of the Kirchhoff equation, Comm. Pure Appl. Math. 47 (1994), 1005-1029.

[9] P. D'Ancona and S. Spagnolo, Kirchhoff type equations depending on a small parameter, Chin. Ann. of Math. 16B (1995), 413-430.

[10] N. Dunford and J.T. Schwartz, Linear Operators, Part I, Wiley-Intersciences, 1988.

[11] M. Ghisi and M. Gobbino, Kirchhoff equations in generalized Gevrey spaces: local existence, global existence, uniqueness, Rend. Istit. Mat. Univ. Trieste 42 (2010), supp., 89-110.

[12] M. Ghisi and M. Gobbino, Kirchhoff equation from quasi-analytic to spectral-gap data, Bull. London Math. Soc. 43 (2011), 374-385.

[13] M. Ghisi and M. Gobbino, Derivative loss for Kirchhoff equations with non-Lipschitz nonlinear term, Ann. Sc. Norm. Super. Pisa Cl. Sci. (5) 8 (2009), no. 4, 613-646.

[14] J.M. Greenberg and S.C. Hu, The initial-value problem for a stretched string, Quart. Appl. Math. 38 (1980), 289-311.

[15] C. Heiming (=C. Kerler), Mapping properties of generalized Fourier transforms and applications to Kirchhoff equations, NoDEA Nonlinear Differential Equations Appl. 7 (2000), 389-414.

[16] F. Hirosawa, Degenerate Kirchhoff equation in ultradifferentiable class, Nonlinear Anal., Ser. A: Theory Methods, 48 (2002), 77-94.

[17] F. Hirosawa, Global solvability for Kirchhoff equation in special classes of non-analytic functions, J. Differential Equations 230 (2006), 49-70.

[18] K. Kajitani, The global solutions to the Cauchy problem for multi-dimensional Kirchhoff equation, Advance in Phase Space Analysis of Partial Differential Equations, (A. Bove, D. Del Santo and M. K. V. Murthy eds.), Progress in Nonlinear Differential Equations and Their Applications, vol. 78, Birkhäuser, Boston 2009, pp. 141-153.

[19] K. Kajitani and K. Yamaguti, On global analytic solutions of the degenerate Kirchhoff equation, Ann. Scuola Norm. Sup. Pisa Cl. Sci. (4) 21 (1994), 279-297.

[20] G. Kirchhoff, Vorlesungen über Mechanik, Teubner, Leibzig, 1876.

[21] T. Kotake and M. Narasimhan, Regularity theorems for fractional powers of a linear elliptic operator, Bull. Soc. Math. France 90 (1962), 449-471.

[22] R. Manfrin, On the global solvability of symmetric hyperbolic systems of Kirchhoff type, Discrete Contin. Dynam. Systems 3 (1997), 91-106.

[23] R. Manfrin, On the global solvability of Kirchhoff equation for non-analytic initial data, J. Differential Equations 211 (2005), 38-60.

[24] T. Matsuyama, Global well-posedness for the exterior initial-boundary value problem to the Kirchhoff equation, J. Math. Soc. Japan 64 (2010), 1167-1204.

[25] T. Matsuyama, The Kirchhoff equation with global solutions in unbounded domains, Rend. Istit. Mat. Univ. Trieste. 42 (2010), suppl., 125-141.

[26] T. Matsuyama and M. Ruzhansky, Global well-posedness of the Kirchhoff equation and Kirchhoff systems, Analytic Methods in Interdisciplinary Applications, Springer Proceedings in Mathematics \& Statistics, Vol. 116, Springer, 2015.

[27] T. Matsuyama and M. Ruzhansky, Scattering for strictly hyperbolic systems with time-dependent coefficients, Math. Nachr. 286 (2013), 1191-1207.

[28] T. Matsuyama and M. Ruzhansky, Global well-posedness of Kirchhoff systems, J. Math. Pures Appl. 100 (2013), 220-240.

[29] K. Mochizuki, Spectral and Scattering Theory for Second Order Elliptic Differential Operators in an Exterior Domain, Lecture Notes Univ. Utah, Winter and Spring 1972. 
[30] T. Nishida, A note on the nonlinear vibrations of the elastic string, Mem. Fac. Engrg. Kyoto Univ. 33 (1971), 329-341.

[31] K. Nishihara, On a global solution of some quasilinear hyperbolic equation, Tokyo J. Math. 7 (1984), 437-459.

[32] S.I. Pohožhaev, On a class of quasilinear hyperbolic equations, Math. USSR Sb. 25 (1975), $145-158$.

[33] R. Racke, Generalized Fourier transforms and global, small solutions to Kirchhoff equations, Asymptotic Analysis 58 (1995), 85-100.

[34] W. Rzymowski, One-dimensional Kirchhoff equation, Nonlinear Analysis 48 (2002), 209-221.

[35] N.A. Shenk II, Eigenfunction expansions and scattering theory for the wave equation in an exterior region, Arch. Rational Mech. Anal. 21 (1966), 120-150.

[36] C.H. Wilcox, Scattering Theory for the D'Alembert Equation in Exterior Domains, Lecture Notes Math. 442 (1975).

[37] T. Yamazaki, Scattering for a quasilinear hyperbolic equation of Kirchhoff type, J. Differential Equations 143 (1998), 1-59.

[38] T. Yamazaki, Global solvability for the Kirchhoff equations in exterior domains of dimension larger than three, Math. Methods Appl. Sci. 27 (2004), 1893-1916.

[39] T. Yamazaki, Global solvability for the Kirchhoff equations in exterior domains of dimension three, J. Differential Equations 210 (2005), 290-316.

TOKio Matsuyama:

Department of Mathematics

Chuo University

1-13-27, Kasuga, BunKyo-KU

TOKYO 112-8551

JAPAN

E-mail address tokio@math.chuo-u.ac.jp

Michael Ruzhansky:

Department of Mathematics

IMPERIAL COLlege LONDON

180 QueEn's Gate, London SW7 2AZ

UNITED KINGDOM

E-mail address m.ruzhansky@imperial.ac.uk 